\title{
How Can We Define Recovery after Acute Kidney Injury? Considerations from Epidemiology and Clinical Trial Design
}

\author{
John A. Kellum \\ The Center for Critical Care Nephrology, CRISMA (Clinical Research, Investigation, and Systems Modeling of Acute Illness) \\ Center, Department of Critical Care Medicine, University of Pittsburgh School of Medicine, Pittsburgh, Pa., USA
}

\section{Key Words}

Acute kidney injury $\cdot$ Biomarkers $\cdot$ Epidemiology $\cdot$ Renal replacement therapy

\begin{abstract}
The recent recognition that acute kidney injury (AKI) may lead to the development of chronic kidney disease and endstage renal disease, with the attendant increase in mortality, has led to interest in the clinical epidemiology and the mechanistic understanding of renal recovery after an episode of AKI. At present, no uniform definition for recovery after AKI exists and various considerations should be taken into account in the development of a definition. Renal recovery after an AKI episode may affect clinical decision making around the initiation of renal replacement therapy, and has significant implication for biomarker assessment and identification of mechanistic targets to guide potential future clinical trials.

(c) 2014 S. Karger AG, Basel
\end{abstract}

(c) 2014 S. Karger AG, Basel

$1660-2110 / 14 / 1274-0081 \$ 39.50 / 0$

\section{Introduction}

Standard definitions for acute kidney injury (AKI) have only been available for a relatively short time $[1,2]$, and yet they have been almost universally integrated into clinical research - though their adoption into clinical practice, while recommended by some [3], has not been seamless $[4,5]$. At the time the Acute Dialysis Quality Initiative (ADQI) proposed the Risk, Injury, Failure, Loss of Kidney Function and End-Stage Renal Disease (RIFLE) criteria for AKI, they also proposed criteria for recovery [1] (table 1). Various studies over the ensuing years have defined recovery differently, although almost all have focused on patients receiving renal replacement therapy (RRT), and at least part of the assessment for recovery involves the ongoing need for dialysis. Indeed, the ADQI criteria had exactly this population in mind.

Targeting Recovery from Acute Kidney Injury: Round Table Conference at the 19th International Conference on Continuous Renal Replacement Therapies (Manchester Grand Hyatt, San Diego, Calif., USA, March 2-3, 2014).

\section{KARGER}

E-Mail karger@karger.com www.karger.com/nec
John A. Kellum, MD, MCCM

Bioengineering, and Clinical and Translational Science 604, Scaife Hall, 3550 Terrace Street Pittsburgh, PA 15261 (USA)

E-Mail kellumja@ccm.upmc.edu 

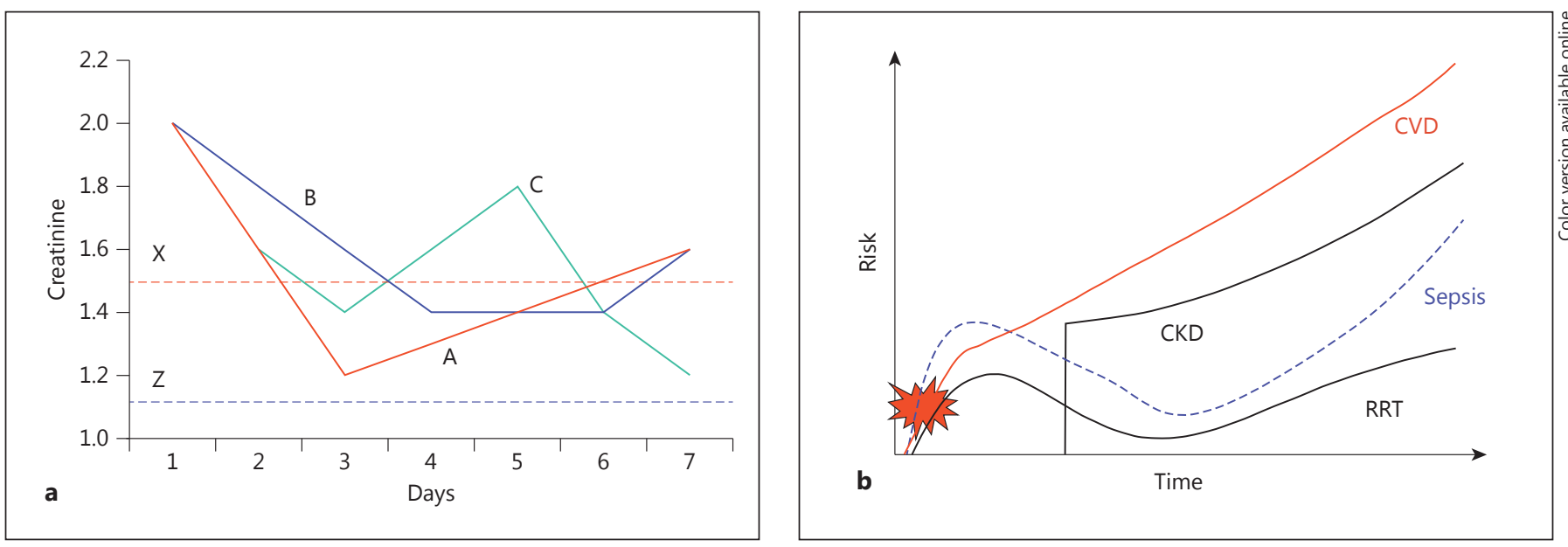

Fig. 1. Short- and long-term recovery. a Various 'recovery patterns' for serum creatinine. Curves A-C represent individual patients; dashed lines $\mathrm{X}$ and $\mathrm{Z}$ represent recovery thresholds. $\mathbf{b}$ Risk profile over time for various outcomes. $\mathrm{CVD}=$ Cardiovascular disease.

Table 1. Definitions for recovery after AKI

\begin{tabular}{|c|c|c|c|c|}
\hline & ADQI [1] 2004 & ATN Trial [25] 2008 & Srisawat et al. [19] 2011 & KDIGO [3] 2012 \\
\hline Complete recovery & $\begin{array}{l}\text { Return to within } 50 \% \\
\text { of baseline serum } \\
\text { creatinine }\end{array}$ & $\begin{array}{l}\text { Return to within } \\
0.5 \mathrm{mg} / \mathrm{dl} \text { of baseline } \\
\text { creatinine }\end{array}$ & $\begin{array}{l}\text { Alive, no RRT, less than } \\
\text { RIFLE-F criteria }\end{array}$ & $\begin{array}{l}\text { GFR } \\
\geq 60 \mathrm{ml} / \mathrm{min} / 1.73 \mathrm{~m}^{2}\end{array}$ \\
\hline Partial recovery & $\begin{array}{l}\text { Off RRT but failed } \\
\text { to return to within } \\
50 \% \text { of baseline serum } \\
\text { creatinine }\end{array}$ & $\begin{array}{l}\text { Off } \mathrm{RRT}^{\mathrm{a}} \text { but failed to } \\
\text { return to within } 0.5 \mathrm{mg} / \\
\text { dl of baseline creatinine }\end{array}$ & & $\begin{array}{l}\text { Off RRT but GFR } \\
<60 \mathrm{ml} / \mathrm{min} / 1.73 \mathrm{~m}^{2} \text { for } \\
<90 \text { days }^{\mathrm{b}}\end{array}$ \\
\hline Non-recovery & $\begin{array}{l}\text { Persistent RRT - } \\
\text { RIFLE-L or -E }\end{array}$ & $\begin{array}{l}\text { Persistent RRT - } \\
\text { RIFLE-L or -E }\end{array}$ & $\begin{array}{l}\text { RIFLE-F, -L or -E at } \\
\text { hospital discharge, } \\
\text { death or RRT }\end{array}$ & $\begin{array}{l}\text { GFR } \\
<60 \mathrm{ml} / \mathrm{min} / 1.73 \mathrm{~m}^{2} \text { for } \\
\geq 90 \text { days or persistent RRT }\end{array}$ \\
\hline
\end{tabular}

a In the ATN trial, patients with a 6-hour creatinine clearance $>20 \mathrm{ml} / \mathrm{min}$ were trialed off RRT, whereas patients with a creatinine clearance $<12 \mathrm{ml} / \mathrm{min}$ had RRT continued [25].

$\mathrm{b}$ The concept of AKD was introduced in the guideline as a way of classifying patients who did not recover after AKI but prior to 90 days when the CKD definitions apply.

Clearly, patients who develop severe AKI (e.g. stage 3) but do not receive RRT cannot be considered to have recovered (even partially) if they do not improve renal function but never require RRT. Conversely, it does not seem appropriate to consider a patient with stage $1 \mathrm{AKI}$ who never improves to have 'no recovery' while a patient with stage $3 \mathrm{AKI}$ improves only to a level equivalent to stage 2 to be 'recovered'. Indeed, as shown in figure 1a, a given patient may have multiple post-AKI trajectories and while being 'free of dialysis' is an important out- come, being left with chronic kidney disease (CKD) is also a concern. In addition, the arbitrary cutoff of 90 days for diagnosis of CKD should by no means trivialize persistent renal dysfunction following AKI just because 90 days has not been reached.

To address the issue of the 'black hole' between AKI and $\mathrm{CKD}$, the Kidney Disease: Improving Global Outcomes (KDIGO) AKI Workgroup proposed the concept of acute kidney disease (AKD). AKD, defined as a glomerular filtration rate (GFR) $<60 \mathrm{ml} / \mathrm{min} / 1.73 \mathrm{~m}^{2}$ or evi- 
dence of structural kidney damage for $<3$ months, provides an operationally integrated bridge between AKI and CKD. The AKD concept, which incorporates the concept of partial renal recovery, might also help raise awareness and engender the necessary clinical mechanisms to follow AKI survivors for progression to $\mathrm{CKD}$, which has been recently highlighted as a missed opportunity for adequate transitions of care $[6,7]$. Macedo et al. [8] were the first to evaluate this formally and found in a small cohort $(\mathrm{n}=$ 84 ) of AKI survivors that $81 \%$ had AKD at hospital discharge; $64 \%$ of patients eventually recovered, though for $17 \%$ this took more than 1 year.

Finally, as illustrated in figure $1 \mathrm{~b}$, a number of nonrenal outcomes can also occur following AKI. To the extent that a patient may appear to recover renal function within a given time frame, we may be tempted to define recovery solely with respect to the kidney and yet if recovery is delayed and non-renal morbidities occur, it is important to capture these aspects as well. When one examines the long-term consequences of AKI, both kidney-specific outcomes (e.g. dialysis and CKD) as well as nonspecific outcomes (e.g. death, cardiovascular disease and recurrent infection) should be considered. However, inclusion of nonspecific outcomes can be problematic when their association with AKI is less strong. The composite endpoint of death, dialysis and persistent renal dysfunction has been referred to as major adverse kidney events (MAKE) [9]. Given that death is actually more common than dialysis after AKI, its inclusion in a composite endpoint is practically mandatory. Non-renal events, especially cardiovascular events, have been the subject of a growing number of publications [10-12].

\section{What Is Recovery from AKI?}

\section{Short- versus Long-Term Outcomes}

The assessment of recovery actually begins at the same time as the assessment of AKI. Patients who progress to more severe stages of AKI are, in fact, failing to recover acutely. By contrast, a patient who experiences a rapid increase in creatinine and then a 'leveling off' is actually experiencing an improvement in function. If we could measure GFR in real time in such a patient, we might observe that GFR is very low or perhaps even zero and then, as function begins to recover, the effect on creatinine is to reduce the rate of increase. Thus, some patients observed to have AKI that 'does not progress' are actually exhibiting partial recovery. Thus, the first short- term recovery question is whether the patient will continue to get worse or not. The most practical, and possibly most patient-centered, aspect of this question is whether the patient will recover enough kidney function to avoid dialysis. However, it is also important to understand this short-term recovery for clinical decision making because patients who progress to more severe stages [13] or remain in a given stage longer [14] have significantly worse clinical outcomes. We do not yet know whether mitigation of progression or persistence of AKI will improve these outcomes or whether the die is cast once injury occurs. However, it seems quite likely that some, if not most, AKI is reversible early in its course since most exposures (e.g. drugs and sepsis) are not instantaneous.

Once the episode of AKI has unfolded and function has reached some degree of steady state, the next step would be to focus on patients who survive an AKI episode and assess the long-term consequences. The fact that episodes of AKI are associated with more rapid progression to CKD in adult patients is a relatively recent observation [15]; the 2008 United States Renal Data System Report revealed that adults with an AKI episode during hospitalization have an approximately 10 -fold greater risk of progressing to end-stage renal disease than patients who did not experience AKI [16]. Similar observations were also reported around the same time for cardiac surgery patients [17]. Thus, short-term recovery endpoints are also candidates as surrogates for these long-term outcomes.

\section{Long-Term Outcomes}

Based on recommendations from the AKI Network [9], the report from the 2011 Workshop of the National Institute of Diabetes and Digestive and Kidney Diseases on Clinical Trial Design [18] proposed a composite endpoint of death, dialysis provision, and incomplete renal recovery at 28 or 60 days. We had already applied this concept in the evaluation of plasma neutrophil gelatinase-associated lipocalin as a predictor of renal recovery defined by the composite of mortality, persistence of RIFLE-F, or need for RRT in patients developing AKI in the setting of community-acquired pneumonia [19]. More recently, we operationalized this endpoint 30 days after AKI (MAKE30) as death, dialysis, or a persistent creatinine level greater than or equal to twice baseline in a study evaluating novel AKI biomarkers [20]. An important consequence of these composite endpoints is that the issue of competing risk between persistent renal dysfunction, RRT, and death is obviated by combining these out- 
comes into a single endpoint. In addition, these endpoints are perhaps more patient centered since kidneys that recover in patients that die will be valued less by these patients [6]. Two of the three components of MAKE (death and dialysis) are unambiguous, although even dialysis may be open to interpretation as to whether it refers to any use of RRT over the observation period versus ongoing use of RRT at the time point studied. Also, patients receiving renal transplants have presumably met the endpoint. The third component of MAKE, persistent renal dysfunction, has not been codified. Many of the issues discussed below for short-term recovery endpoints may also pertain to this component of MAKE.

Finally, a recent study by Chawla et al. [11] examined a composite endpoint that incorporated both renal (MAKE) and cardiovascular endpoints. Major adverse renocardiovascular events (MARCE) include MAKE endpoints along with cardiovascular events. In an analysis of nearly 40,000 patients, the authors found that patients with AKI had worse major adverse renocardiovascular events compared to those who had a myocardial infarction without AKI [adjusted hazard ratio, 1.37 (95\% confidence interval 1.32-1.42)]. Patients with both AKI and myocardial infarction had the highest risk [adjusted hazard ratio, 1.92 (95\% confidence interval 1.86-1.99)] [11].

It should also be noted, however, that so-called longterm endpoints can themselves be misleading. AKI may well effect outcomes many years later. Murugan et al. [21] found evidence of both early (up to 90 days) effects of AKI on survival as well as late (after 9 months) effects in patients with AKI in the setting of pneumonia. The Acute Renal Failure Trial Network Study (ATN) investigators found a similar bimodal association with mortality in hospital survivors in that trial - an acute phase out to day 42 and a late phase after day 148 [22]. Macedo et al. [8] found that GFR did not stabilize in some patients after AKI until 18 months [8]. Recently, Linder et al. [23] reported that even patients with stage 1 AKI have an increased risk of death 10 years later. Thus, we cannot judge recovery endpoints solely on how well they are surrogates for 90-day or even 1-year outcomes when the hazards of non-recovery may well extend more than a decade into the future.

\section{Creatinine-Based Definitions}

Used for either short- or long-term outcomes, a change in creatinine itself reflects late functional changes which are inherently delayed since the kidneys contain the innate ability to maintain function by hyperfiltration and compensatory hypertrophy of the remaining healthy nephrons, irrespective of the etiology of the kidney damage. This compensation allows for continued clearance of plasma solutes, so that serum creatinine increases only after about $50 \%$ of nephrons have been lost. Thus, it is important to note that even if creatinine-based GFR returns to normal, there may be a potential loss of renal reserve, so that repeated episodes of AKI may unmask clinically detectable CKD.

Alternatives to serum creatinine-based definitions of renal recovery have been reviewed elsewhere [6]. These include timed urine collections for creatinine clearance measurement, direct GFR assessment by radionuclide clearance and GFR estimation using cystatin C, a molecule that has ideal properties of clearance by filtration with a serum concentration that is not dependent on muscle mass. In the future, clinical research may employ these methods to better assess recovery and functional reserve and to help reduce the sole reliance on serum creatinine.

\section{Considerations in Defining Recovery from AKI}

\section{Defining Short-Term Recovery}

Whether we are examining short-term outcomes for their own sake or whether we are interested in understanding what they tell us about long-term outcomes, several issues for ascertaining recovery must be settled. These issues relate to how we operationalize the various dimensions of recovery and can be grouped into four domains: inception; magnitude; timing, and confounding. Unlike for long-term outcome where consensus is forming around the MAKE composite [18], presently, there is insufficient evidence to advocate a specific definition for short-term recovery following AKI. However, understanding the various dimensions of recovery will allow us to develop definitions that can be tested and, once validated, enter the standard operating procedure for AKI clinical research in the same way that the definitions of AKI already have. But first, we must define the terms of recovery.

\section{Terminology}

The terms complete and partial recovery were proposed by ADQI in the context of acute dialysis. The concept of complete recovery as a return to 'normal' or 'baseline' seems strongly grounded in medicine and has high construct validity. It is usually defined by the absence of disease, and when proposed along with the RIFLE crite-
Kellum 
ria by ADQI, it was defined as a return to creatinine less than the threshold for RIFLE-R or $50 \%$ of baseline. Thus, a patient who has a baseline creatinine of $1.0 \mathrm{mg} / \mathrm{dl}$ who recovers to a creatinine $<1.5 \mathrm{md} / \mathrm{dl}$ would be said to have complete recovery. Whether or not a different threshold should be chosen, the concept of complete recovery seems logical. Partial recovery, similarly, would cover the middle ground between complete and no recovery. In the context of acute dialysis, non-recovery is usually defined as death or 'permanent dialysis', so partial recovery in our example patient would be defined as the absence of dialysis but with a creatinine $\geq 1.5 \mathrm{mg} / \mathrm{dl}$. However, outside the context of dialysis, non-recovery and therefore partial recovery would seem to require some other features to establish whether some improvement did or did not occur.

The second set of terms that are needed to define recovery concern timing. There are three times that are important in this assessment. The first is the time when recovery begins; the second is the time when it can be defined, and the third is the time when it is measured. The starting time for recovery is obviously also the end time for an episode of AKI (or at least the start of the end). Because there are normal fluctuations, similar to economics, recovery (or recession) can only be defined once it is sustained (for two quarters in the case of the economy) and then it is said to have occurred at the beginning of the first period where the change took place. For the purposes of defining recovery of renal function, the term sustained can also be used. A recovery that is sustained for more than a specific number of days, for example, might qualify as a 'true' recovery and then if there is subsequent deterioration it is viewed as a new episode of AKI. It is important to set the duration of recovery long enough that brief fluctuations (like those in the market) do not trigger the definition, yet not so long as to be impractical or difficult to ascertain. Because there is both a duration of AKI and a duration of recovery, the term persistent is better suited to quantify the duration of AKI and to distinguish it from the term sustained, which is used to quantify the duration of recovery.

\section{Inception: Starting Point for Recovery}

The starting point for recovery refers to both time when recovery assessment can begin and the state of AKI at that point. There is a vast difference between recovery from a small change in renal function (e.g. stage 1) and recovery from more severe forms (e.g. on RRT). It is unlikely that a single definition for recovery will make sense across all severities of AKI while standard definitions are more likely to work when applied to patients with similar disease severity. Of course, this problem is not unique to AKI. What constitutes recovery in the context of a cold is very different than in the context of cancer. However, this may only be a concern for defining non-recovery or partial recovery since the concept of 'disease free' as a complete recovery may still hold regardless of the starting point. Nevertheless, the more homogenous the population is from which recovery is being assessed, the more straightforward the recovery assessment will be.

Interestingly, this problem of the starting state also involves the application of the AKI definitions themselves. If a given patient meets the criteria for AKI on urine output first (which is common), how much time should be given before we can be confident that a serum creatinine change will not follow? If we are looking at recovery from stage 2 or greater AKI, how should we interpret changes in the second criterion (urine output or creatinine) when the other criterion was used to make the initial diagnosis? Said another way, how persistent is the episode of AKI and how sustained does recovery need to be when both criteria are changing in different time courses? All of these questions need to be considered in the development of a definition for AKI.

\section{Magnitude of Recovery}

As seen in table 1, recovery may be assessed as a relative or absolute change, or as a fixed threshold. There are advantages and disadvantages to each approach. A fixed threshold has face validity because one can set the threshold at a level of function that was used to define disease and therefore recovery is defined by no longer having disease. The most commonly used fixed threshold is GFR $\geq 60 \mathrm{ml} / \mathrm{min} / 1.73 \mathrm{~m}^{2}$ because anything below this level is defined as CKD. The problem is that patients will have different baselines and thus one has to either exclude patients who are ineligible for this threshold because their baseline function is already worse or a different threshold must be used. Another approach is to use a relative threshold - relative to baseline function. ADQI recommended a recovery threshold of $50 \%$ above baseline creatinine for complete recovery. One of the few studies to examine various thresholds was a recent study by Pannu et al. [24] who found that a recovery threshold $<35 \%$ of baseline did not have any relationship with mortality and this relationship only became significant when $>55 \%$. However, for end-stage renal disease or persistence of creatinine twice baseline, a $25 \%$ or greater threshold was associated with an increased risk. However, although the study was of reasonable size, the individual thresholds were not well 
represented and the confidence limits around the hazard ratios were wide.

Furthermore, regardless of which threshold is set, there will always be some patients on just one side or the other. This problem is compounded by the issue of the starting point discussed above. For example, if we set a threshold of GFR $\geq 60 \mathrm{ml} / \mathrm{min} / 1.73 \mathrm{~m}^{2}$, a patient who has a baseline GFR of 70, develops AKI, and reaches 30 but recovers to 59 will be classified as a non-recoverer. While a patient with a baseline GFR of 100 who reaches 55 with AKI and only recovers to $62 \mathrm{ml} / \mathrm{min} / 1.73 \mathrm{~m}^{2}$ will be classified as recovering. Defining recovery on the basis of fixed or relative improvement in GFR or creatinine is problematic for different reasons. Recovery from very severe AKI (e.g. GFR $10 \mathrm{ml} / \mathrm{min} / 1.73 \mathrm{~m}^{2}$ ) might be dramatic (e.g. a 300\% improvement) but still leave the patient with severely decreased function, while smaller improvements in less severe cases might still achieve near-normal function. These problems are attenuated if the starting points are more homogenous but a great deal of consideration is needed if one defines recovery by the magnitude of functional improvement rather than in relation to some predefined benchmark.

Part of this problem concerns the tension between clinical relevance, which is mainly concerned with the degree of function that one ends up with, and biological relevance, which is more likely tied to relative numbers of surviving cells or functioning nephrons. Combinations of fixed and relative changes may also be considered (e.g. $>100 \%$ increase in GFR and final GFR $>30 \mathrm{ml} / \mathrm{min} /$ $1.73 \mathrm{~m}^{2}$ ), and this approach may offer the best balance of clinical and biological relevance.

\section{Timing of Recovery}

Timing is a critical dimension of recovery. Timing can refer to how persistent an episode of AKI is or how sustained the recovery is. It may also refer to the time when recovery is assessed. How long we wait after renal function starts to improve before we can measure recovery is in some ways linked to how sustained recovery has to be in order to fulfill our criteria. Consider the extreme case in which one considers a single creatinine as sufficiently sustained to define recovery. If the creatinine is ever observed to meet the level set for recovery, we would classify this as recovery if it were to deteriorate immediately thereafter. This approach obviously risks false attribution of recovery in the setting of small fluctuations in renal function. Conversely, if one requires, say a year, of sustained recovery, then multiple episodes of AKI, each followed by improvement in function, would be falsely bundled into a single event. What we seek is a definition for recovery that is sustained enough to have some confidence that it is real and yet not require an unreasonably long time frame to assess for it. Finally, it is possible that we might require a different threshold for sustained recovery when the episode of AKI is short than when it is very persistent.

\section{Confounding}

The final domain to consider is what to do when outside factors influence our ability to ascertain recovery. Broadly speaking, these confounders fit into two categories: competing endpoints (e.g. death and dialysis) and interference. I have already mentioned competing endpoints in the consequence of long-term outcomes and the role of composite endpoints as a way to deal with this problem. For shortterm recovery assessment, the same rules generally apply. One can only apply a creatinine-based rule set to patients who are alive and off dialysis. However, what happens when a patient meets criteria for recovery and subsequently dies? From a patient-centered perspective, this is not recovery and yet there may have been biological recovery prior to a death caused by something else. Again, timing is in play. If the death and the recovery are remote (e.g. weeks or more apart), it may be more reasonable to separate them and allow for classification of recovery without considering subsequent mortality.

Interference by contrast refers to anything that impedes the ascertainment of recovery. Loss of muscle mass may reduce creatinine without changing GFR; severe liver disease and some drugs interfere with creatinine measurement; open abdomens with large ascites losses may result in extrarenal 'clearance' of creatinine, and large volumes of intravenous fluids or massive blood transfusions may dilute creatinine. Most of these problems can be addressed by alternative methods to assess GFR but only when planned for. A more common and difficult problem is when data are missing, often because patients are discharged or lost to follow-up. This may produce a situation of informative censoring because patients who are healthier and therefore more likely to recover may be more likely to be discharged prior to complete recovery. Careful considerations are necessary to avoid bias in ascertaining recovery in such patients.

\section{Final Remarks and Recommendations}

Like for AKI itself, standard definitions for recovery would facilitate clinical research. Unlike AKI onset, few studies have focused on recovery and most of these have
Kellum 
only focused on recovery from RRT. It is important to assess recovery after AKI in both short and long term. For long-term assessment, there is a strong rationale to use a MAKE composite which includes death, dialysis, and persistent renal dysfunction (e.g. twice baseline creatinine). For short-term recovery, there is no consensus as to how to define recovery. However, the following recommendations can be made.

(1) Recovery is context dependent - recovery from RRT is not the same as recovery from stage 1 .

(2) The concept of complete recovery as return to baseline or a normal or 'disease-free' state has a strong clinical and biological rationale. A threshold for what is considered normal or an acceptable departure from baseline needs to be determined.

(3) The dimensions of recovery that require standardization include inception - under what context and from what condition does recovery begin; magnitude - how much recovery and to what level of function is required, and timing - when does recovery assessment start and how long does it need to be sustained.

(4) Consideration should be given to potential confounding - either by competing endpoints such as death or dialysis or through interference in creatinine measurement or other measures of recovery.

\section{Conclusions}

Recovery of renal function following an episode of AKI is far from certain and a growing body of evidence has established that progression of underlying CKD or development of de novo CKD may occur following an episode of AKI. Patients who fail to recover will have a significantly shorter life span and succumb to a number of morbidities. The underlying cellular and physiologic mechanisms that drive outcomes after AKI are poorly characterized. Epidemiologic evidence strongly suggests that advanced age is the most significant risk factor for non-recovery of renal function, but this may merely reflect a diminished renal functional reserve in this population. More research is needed, and paramount to the conduct of this investigation is a standard framework for assessing recovery. This review attempts to put on the table the various considerations that are needed in developing definitions for recovery following AKI.

\section{References}

1 Bellomo R, Ronco C, Kellum JA, Mehta RL, Palevsky P: Acute renal failure - definition, outcome measures, animal models, fluid therapy and information technology needs: the Second International Consensus Conference of the Acute Dialysis Quality Initiative (ADQI) Group. Crit Care 2004;8:R204-R212.

-2 Mehta RL, Kellum JA, Shah SV, Molitoris BA, Ronco C, Warnock DG, Levin A: Acute Kidney Injury Network: report of an initiative to improve outcomes in acute kidney injury. Crit Care 2007;11:R31.

-3 Kidney Disease: Improving Global Outcomes (KDIGO) Acute Kidney Injury Work Group: KDIGO Clinical Practice Guideline for Acute Kidney Injury. Kidney Int Suppl 2012; 2:1138.

4 Palevsky PM, Liu KD, Brophy PD, Chawla LS, Parikh CR, Thakar CV, et al: KDOQI US commentary on the 2012 KDIGO clinical practice guideline for acute kidney injury. Am J Kidney Dis 2013;61:649-672.

5 The ad-hoc working group of ERBP, Fliser D, Laville M, Covic A, Fouque D, Vanholder R, et al: A European Renal Best Practice (ERBP) position statement on the Kidney Disease Improving Global Outcomes (KDIGO) clinical practice guidelines on acute kidney injury: part 1: definitions, conservative management and contrast-induced nephropathy. Nephrol Dial Transplant 2012;27:4263-4272.
6 Goldstein SL, Chawla L, Ronco C, Kellum JA: Renal recovery. Crit Care 2014;18:301.

7 Goldstein SL, Jaber BL, Faubel S, Chawla LS; Acute Kidney Injury Advisory Group of American Society of Nephrology: AKI transition of care: a potential opportunity to detect and prevent CKD. Clin J Am Soc Nephrol 2013;8:476-483.

-8 Macedo E, Zanetta DMT, Abdulkader RCRM: Long-term follow-up of patients after acute kidney injury: patterns of renal functional recovery. PLoS One 2012;7:e36388.

-9 Shaw A: Models of preventable disease: contrast-induced nephropathy and cardiac surgery-associated acute kidney injury. Contrib Nephrol 2011;174:156-162.

-10 Murugan R, Weissfeld L, Yende S, Singbartl K, Angus DC, Kellum JA, et al: Association of statin use with risk and outcome of acute kidney injury in community-acquired pneumonia. Clin J Am Soc Nephrol 2012;7:895905.

11 Chawla LS, Amdur RL, Shaw AD, Faselis C, Palant CE, Kimmel PL: Association between $\mathrm{AKI}$ and long-term renal and cardiovascular outcomes in United States veterans. Clin J Am Soc Nephrol 2014;9:448-456.

12 Wu V-C, Wu C-H, Huang T-M, Wang C-Y, Lai C-F, Shiao C-C, et al: Long-term risk of coronary events after AKI. J Am Soc Nephrol 2014;25:595-605.
13 Hoste EAJ, Clermont G, Kersten A, Venkataraman R, Angus DC, De Bacquer D, et al: RIFLE criteria for acute kidney injury are associated with hospital mortality in critically ill patients: a cohort analysis. Crit Care 2006;10:R73.

14 Coca SG, King JT, Rosenthal RA, Perkal MF, Parikh CR: The duration of postoperative acute kidney injury is an additional parameter predicting long-term survival in diabetic veterans. Kidney Int 2010;78:926-933.

15 Thakar CV, Christianson A, Himmelfarb J, Leonard AC: Acute kidney injury episodes and chronic kidney disease risk in diabetes mellitus. Clin J Am Soc Nephrol 2011;6:25672572.

-16 Collins AJ, Foley RN, Herzog C, Chavers B, Gilbertson D, Ishani A, Kasiske B, Liu J, Mau LW, McBean M, et al: United States Renal Data System 2008 Annual Data Report. Am J Kidney Dis 2009;53(suppl 1):S1-S374.

-17 Hobson CE, Yavas S, Segal MS, Schold JD, Tribble CG, Layon AJ, Bihorac A: Acute kidney injury is associated with increased longterm mortality after cardiothoracic surgery. Circulation 2009;119:2444-2453.

18 Palevsky PM, Molitoris BA, Okusa MD, Levin A, Waikar SS, Wald R, Chertow GM, Murray PT, Parikh CR, Shaw AD, et al: Design of clinical trials in acute kidney injury: report from an NIDDK workshop on trial methodology. Clin J Am Soc Nephrol 2012;7:844-850. 
19 Srisawat N, Wen X, Lee M, Kong L, Elder M, Carter M, Unruh M, Finkel K, Vijayan A, Ramkumar M, et al: Urinary biomarkers and renal recovery in critically ill patients with renal support. Clin J Am Soc Nephrol 2011;6: 1815-1823.

-20 Kashani K, Al-Khafaji A, Ardiles T, Artigas A, Bagshaw SM, Bell M, Bihorac A, Birkhahn R, Cely CM, Chawla LS, et al: Discovery and validation of cell cycle arrest biomarkers in human acute kidney injury. Crit Care 2013; $17: R 25$.
1 Murugan R, Karajala-Subramanyam V, Lee M, Yende S, Kong L, Carter M, et al: Acute kidney injury in non-severe pneumonia is associated with an increased immune response and lower survival. Kidney Int 2010;77:527535.

2 Zhang JH, Palevsky PM, Chertow GM, Hartigan J, O'Connor TZ, Guarino P, et al: Piecewise analysis of patient survival after onset of AKI. Clin J Am Soc Nephrol 2013;8:16791684.

23 Linder A, Fjell C, Levin A, Walley KR, Russell JA, Boyd JH: Small acute increases in serum creatinine are associated with decreased long term survival in the critically ill. Am J Respir Crit Care Med 2014;189:1075-1081.
24 Pannu N, James M, Hemmelgarn B, Klarenbach S; Alberta Kidney Disease Network: Association between AKI, recovery of renal function, and long-term outcomes after hospital discharge. Clin J Am Soc Nephrol 2013; 8:194-202.

25 VA/NIH Acute Renal Failure Trial Network, Palevsky PM, Zhang JH, O'Connor TZ, Chertow GM, Crowley ST, et al: Intensity of renal support in critically ill patients with acute kidney injury. N Engl J Med 2008;359:7-20. 